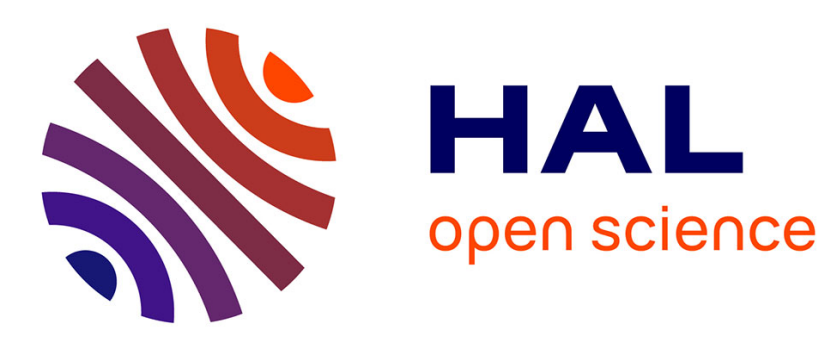

\title{
Enhancement of intermediate mass dimuons in nucleus-nucleus collisions at the CERN-SPS
}

M. Abreu, B. Alessandro, C. Alexa, R. Arnaldi, M. Atayan, C. Baglin, A. Baldit, M. Bedjidian, S. Beole, V. Boldea, et al.

\section{- To cite this version:}

M. Abreu, B. Alessandro, C. Alexa, R. Arnaldi, M. Atayan, et al.. Enhancement of intermediate mass dimuons in nucleus-nucleus collisions at the CERN-SPS. Rencontres du Vietnam 4, Jul 2000, Hanoi, Vietnam. pp.509-518. in2p3-00011619

\section{HAL Id: in2p3-00011619 \\ https://hal.in2p3.fr/in2p3-00011619}

Submitted on 17 Oct 2002

HAL is a multi-disciplinary open access archive for the deposit and dissemination of scientific research documents, whether they are published or not. The documents may come from teaching and research institutions in France or abroad, or from public or private research centers.
L'archive ouverte pluridisciplinaire HAL, est destinée au dépôt et à la diffusion de documents scientifiques de niveau recherche, publiés ou non, émanant des établissements d'enseignement et de recherche français ou étrangers, des laboratoires publics ou privés. 


\title{
ENHANCEMENT OF INTERMEDIATE MASS DIMUONS IN NUCLEUS-NUCLEUS COLLISIONS AT THE CERN-SPS
}

\author{
L. CAPELLI, for the NA50 collaboration
}

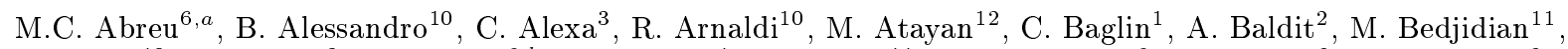
S. Beolè ${ }^{10}$, V. Boldea ${ }^{3}$, P. Bordalo ${ }^{6, b}$, A. Bussière ${ }^{1}$, L. Capelli $^{11}$, L. Casagrande ${ }^{6, c}$, J. Castor $^{2}$, T. Chambon $^{2}$, B. Chaurand ${ }^{9}$, I. Chevrot ${ }^{2}$, B. Cheynis ${ }^{11}$, E. Chiavassa ${ }^{10}$, C. Cicalò ${ }^{4}$, T. Claudino ${ }^{6}$, M.P. Comets ${ }^{8}$, N. Constans ${ }^{9}$, S. Constantinescu ${ }^{3}$, N. De Marco ${ }^{10}$, A. De Falco ${ }^{4}$, G. Dellacasa ${ }^{10, d}$, A. Devaux ${ }^{2}$, S. Dita $^{3}$, O. Drapier ${ }^{11}$, L. Ducroux ${ }^{11}$, B. Espagnon ${ }^{2}$, J. Fargeix ${ }^{2}$, P. Force $^{2}$, M. Gallio ${ }^{10}$, Y.K. Gavrilov ${ }^{7}$, C. Gerschel ${ }^{8}$, P. Giubellino ${ }^{10}$, M.B. Golubeva ${ }^{7}$, M. Gonin ${ }^{9}$, A.A. Grigorian ${ }^{12}$, J.Y. Grossiord ${ }^{11}$, F.F. Guber ${ }^{7}$, A. Guichard ${ }^{11}$, H. Gulkanyan ${ }^{12}$, R. Hakobyan ${ }^{12}$, R. Haroutunian ${ }^{11}$, M. Idzik ${ }^{10, e}$, D. Jouan ${ }^{8}$, T.L. Karavitcheva ${ }^{7}$, L. Kluberg ${ }^{9}$, A.B. Kurepin ${ }^{7}$, Y. Le Bornec ${ }^{8}$, C. Lourenço $^{5}$, P. Macciotta ${ }^{4}$, M. Mac Cormick ${ }^{8}$, A. Marzari-Chiesa ${ }^{10}$, M. Masera ${ }^{10}$, A. Masoni ${ }^{4}$, S. Mehrabyan ${ }^{12}$, M. Monteno ${ }^{10}$, A. Musso ${ }^{10}$, P. Petiau ${ }^{9}$, A. Piccotti ${ }^{10}$, J.R. Pizzi ${ }^{11}$, F. Prino ${ }^{10}$, G. Puddu ${ }^{4}$, C. Quintans ${ }^{6}$, S. Ramos ${ }^{6, b}$, L. Ramello ${ }^{10, d}$, P. Rato Mendes ${ }^{6}$, L. Riccati ${ }^{10}$, A. Romana $^{9}$,

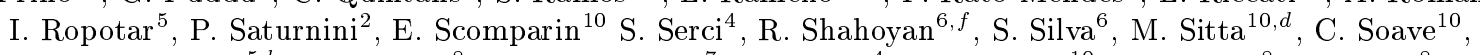
P. Sonderegger ${ }^{5, b}$, X. Tarrago ${ }^{8}$, N.S. Topilskaya ${ }^{7}$, G.L. Usai ${ }^{4}$, E. Vercellin ${ }^{10}$, L. Villatte ${ }^{8}$, N. Willis $^{8}$.

${ }^{1}$ LAPP, CNRS-IN2P3, Annecy-le-Vieux, France.

${ }^{2}$ LPC, Univ. Blaise Pascal and CNRS-IN2P3, Aubière, France.

${ }^{3}$ IFA, Bucharest, Romania.

${ }^{4}$ Università di Cagliari/INFN, Cagliari, Italy.

${ }^{5}$ CERN, Geneva, Switzerland.

${ }^{6}$ LIP, Lisbon, Portugal.

7 INR, Moscow, Russia.

${ }^{8}$ IPN, Univ. de Paris-Sud and CNRS-IN2P3, Orsay, France.

${ }^{9}$ LPNHE, Ecole Polytechnique and CNRS-IN2P3, Palaiseau, France.

${ }^{10}$ Università di Torino/INFN, Torino, Italy.

11 IPN, Univ. Claude Bernard Lyon-I and CNRS-IN2P3, Villeurbanne, France.

12 YerPhI, Yerevan, Armenia.

a) also at UCEH, Universidade de Algarve, Faro, Portugal

b) also at IST, Universidade Técnica de Lisboa, Lisbon, Portugal

c) now at CERN

d) Universitá del Piemonte Orientale, Alessandria and INFN-Torino, Italy

e) now at Faculty of Physics and Nuclear Techniques, University of Mining and Metallurgy, Cracow, Poland f) on leave of absence of YerPhI, Yerevan, Armenia

\begin{abstract}
A 4-Dimensional unfolding method has been applied to data collected in $\mathrm{p}-\mathrm{A}, \mathrm{S}-\mathrm{U}$ and $\mathrm{Pb}-\mathrm{Pb}$ collisions. The intermediate mass dimuon region (IMR) between 1.6 and $2.5 \mathrm{GeV} / \mathrm{c}^{2}$ has been studied. It is shown that the superposition of open charm and Drell-Yan process extrapolated from $\mathrm{p}-\mathrm{A}$ distributions is unable to describe the dimuon yield measured in $\mathrm{S}-\mathrm{U}$ and $\mathrm{Pb}-\mathrm{Pb}$ collisions. From the 450 $\mathrm{GeV} / \mathrm{c} \mathrm{p}-\mathrm{A}$ data, we deduce a charm cross section in agreement with direct measurements of other experiments. A model based on $\mathrm{D}$ and $\overline{\mathrm{D}}$ mesons rescattering is not able to reproduce the experimental spectra. Two possible explanations of the observed excess are discussed.
\end{abstract}

\section{Introduction}

First studies of NA50 data ${ }^{1}$ have shown that the proton-nucleus dimuon mass spectra in the mass range 1.5 to $2.5 \mathrm{GeV} / \mathrm{c}^{2}$ are correctly reproduced by a superposition of Drell-Yan (DY) and open charm (DD) dimuon decays. A linear extrapolation of proton-nucleus sources to nucleus-nucleus collisions underestimates this intermediate mass region data. The nature of this excess, behaving like charm, is not known. The data analysis has been done on a 1-Dimensional basis in each of the dimuon pertinent kinematical variables $\left(M, P_{T}, Y_{c m}\right.$ and $\left.\cos \left(\Theta_{c s}\right)\right)$. However, computing the acceptance in one variable assumes that the distribution functions in the other variables are known and in addition that there are no correlations among them. This hypothesis may not be true for the physical processes at work in the intermediate mass region. Therefore, a more general 4-Dimensional deconvolution technique ${ }^{2}$, has been used to correct the data from the apparatus effects without the need to postulate the presence of specific processes. We present here results obtained using this method on both proton-nucleus and nucleus-nucleus data. Finally, these results are compared with the predictions of some theoretical models. 


\section{Apparatus overview}

The NA50 detector ${ }^{3}$, located in the CERN-SPS North Area, consists mainly of a muon spectrometer which includes a hadron absorber and an air gap toroidal magnet. On both sides of the magnet two sets of four MWPCs are placed for muon tracking. Four scintillator hodoscopes provide the dimuon trigger. Three detectors allow to determine the centrality of the collision: an electromagnetic calorimeter, a multiplicity detector and a zero degree calorimeter.

\section{Analysis}

The 4-Dimensional deconvolution has been applied to the signal distributions obtained after background subtraction. The deconvoluted mass spectra are represented in the mass range 1.6 to $8 \mathrm{GeV} / \mathrm{c}^{2}$ by a sum of the known sources: $\mathrm{J} / \psi$ and $\psi^{\prime}$ resonances together with contributions from the DY process and DD decays:

$$
\frac{\mathrm{dN}}{\mathrm{d} M}=n^{\mathrm{J} / \psi} \frac{\mathrm{dN}^{\mathrm{J} / \psi}}{\mathrm{d} M}+n^{\psi^{\prime}} \frac{\mathrm{dN}^{\psi^{\prime}}}{\mathrm{d} M}+n^{\mathrm{DY}} \frac{\mathrm{dN}^{\mathrm{DY}}}{\mathrm{d} M}+n^{\mathrm{D} \overline{\mathrm{D}}} \frac{\mathrm{dN}^{\mathrm{D} \overline{\mathrm{D}}}}{\mathrm{d} M}
$$

A gaussian shape has been assumed for the $\mathrm{J} / \psi$ and $\psi^{\prime}$ resonances. The shape of the DY and DD dimuon mass distributions has been computed from the event generator PYTHIA 6.1 ${ }^{4}$ with the MRS A set of parton distribution functions and a c quark mass $\mathrm{m}_{\mathrm{c}}=1.5 \mathrm{GeV} / \mathrm{c}^{2}$. The pt distributions have been calculated using $\sigma_{\mathrm{k}_{\mathrm{T}}}=0.8 \mathrm{GeV} / \mathrm{c}$ for Drell-Yan and $\sigma_{\mathrm{k}_{\mathrm{T}}}=1 \mathrm{GeV} / \mathrm{c}$ for DD. Drell-Yan calculations have been corrected for isospin effects. A seven parameter fit on each of the mass spectra gives the normalization of each process.

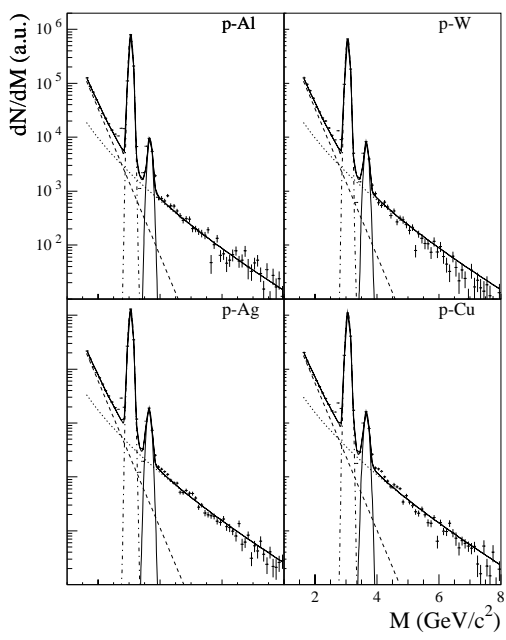

Figure 1: Simultaneous fit of the $4 \mathrm{p}-\mathrm{A}$ mass spectra. The dotted line represents the DY contribution and the dashed line is the DD process.

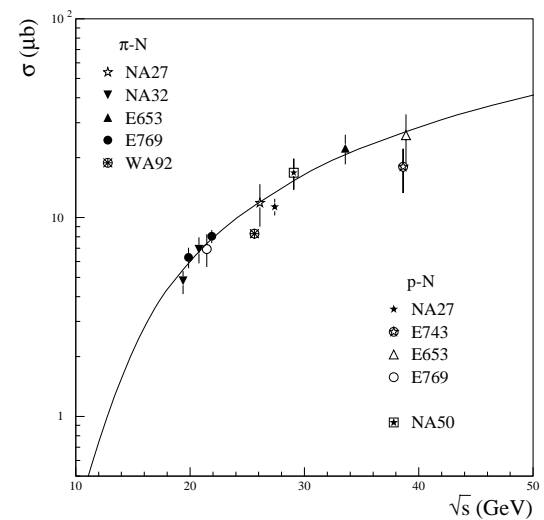

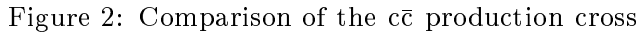
section $\left(\mathrm{x}_{\mathrm{F}}>0\right)$ extracted in this analysis with direct measurements.

\section{$3.1 p-A$ collisions}

This analysis is first applied on four different sets ( $\mathrm{Al}, \mathrm{Cu}, \mathrm{Ag}$ and $\mathrm{W}$ ) of $450 \mathrm{GeV}$ proton-nucleus collisions. The measured data are unfolded in the kinematical domain $\mathcal{D}_{\mathrm{pA}}$, defined by the cuts $M>$ $1.6 \mathrm{GeV} / \mathrm{c}^{2},-0.2<Y_{c m}<0.4$ and $-0.3<\cos \left(\Theta_{c s}\right)<0.3$. Figure 1 shows the result of the simultaneous fit of the four $\mathrm{p}-\mathrm{A}$ data imposing the same ratio $\mathrm{DD} /\left.\mathrm{DY}\right|_{\mathrm{pN}}$ in the intermediate mass region (IMR) $1.6<M<2.5 \mathrm{GeV} / \mathrm{c}^{2}$. The extracted ratio is $\mathrm{D} \overline{\mathrm{D}} /\left.\mathrm{DY}\right|_{\mathrm{pN}, 450}=4.13 \pm 0.15$. From this value, we can estimate the total open charm production cross section $\sigma_{c \bar{c}}$ at $450 \mathrm{GeV} / \mathrm{c}$. From the DY cross section measured in pp collisions ${ }^{5}$ and using PYTHIA, we can deduce the DY cross section in the domain $\mathcal{D}_{\mathrm{pA}}$. After correcting the $\mathrm{D} \overline{\mathrm{D}} / \mathrm{DY}$ ratio by the mean branching ratio of $\mathrm{D}$-mesons into muons, we obtain a total charm production cross section of $\sigma_{\mathrm{c} \overline{\mathrm{c}}}=(35.1 \pm 6) \mu \mathrm{b}$. This value, divided by 2 to take into account only the forward hemisphere, is in agreement with direct measurements, as can be seen in figure 2 . We 
conclude that the IMR spectra in $\mathrm{p}-\mathrm{A}$ collisions are reasonably well described as a superposition of DY and DD dimuons.

\section{2 $S-U$ and $P b-P b$ collisions}

As a next step in the IMR study, the $200 \mathrm{GeV} /$ nucleon $\mathrm{S}-\mathrm{U}$ and $158 \mathrm{GeV} /$ nucleon $\mathrm{Pb}-\mathrm{Pb}$ data have been analysed as a function of centrality bins ( 5 for $\mathrm{S}-\mathrm{U}$ and 7 for $\mathrm{Pb}-\mathrm{Pb}$ ) based on the electromagnetic transverse energy $\mathrm{E}_{\mathrm{T}}$. The measured data have been deconvoluted with the 4-Dimensional method in the following domain $\mathcal{D}_{\mathrm{AB}}: M>1.6 \mathrm{GeV} / \mathrm{c}^{2}, 0.2<Y_{c m}<0.8$ and $-0.3<\cos \left(\Theta_{c s}\right)<0.3$. We first try to describe the mass spectra performing a linear extrapolation on the nuclei masses of the dimuon sources already present in proton induced collisions. The estimated $\mathrm{D} \overline{\mathrm{D}} /\left.\mathrm{DY}\right|_{\mathrm{AB}}$ ratio has been deduced from the proton-nucleon ratio using the energy dependence given by PYTHIA. Taking as normalization the number of high mass DY events $\left(M>4.2 \mathrm{GeV} / \mathrm{c}^{2}\right)$, we find that the sum of the expected sources systematically underestimates the IMR data. The ratio data over expected sources in the mass range 1.6 to $2.5 \mathrm{GeV} / \mathrm{c}^{2}$ has been plotted in figure 3 as a function of the number of participant nucleons $\mathrm{N}_{\mathrm{part}}$. The data exceed the expected sources and this excess grows with the centrality of the collision.

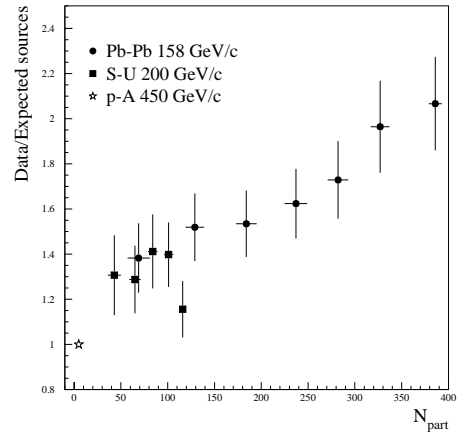

Figure 3: The ratio data over expected sources as a function of $\mathrm{N}_{\text {part }}$.

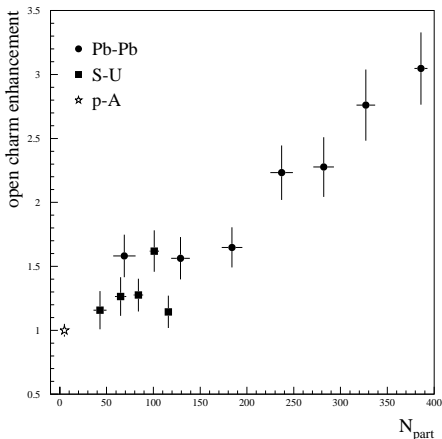

Figure 4: The enhancement of the open charm yield as a function of $\mathrm{N}_{\text {part }}$.

\section{Excess origin}

Several models ${ }^{6-9}$ have been proposed to explain the excess observed in the IMR.

\subsection{Charm enhancement}

Wang and Wong ${ }^{6}$ propose that the observed excess is due to an enhancement of the charm component. To quantify this possible enhancement, the IMR ion mass spectra have been described as a superposition of $\mathrm{DY}$ and $\mathrm{DD}$ and fitted leaving free the normalization constants. The corresponding DD/DY ratios extracted from the fits are then compared to the expected ones deduced from the proton-nucleus ratio. The enhancement factor defined as:

$$
\mathrm{E}=\left(\frac{\mathrm{D} \overline{\mathrm{D}}}{\mathrm{DY}}\right)_{\text {measured }} /\left(\frac{\mathrm{D} \overline{\mathrm{D}}}{\mathrm{DY}}\right)_{\text {expected }}
$$

is plotted in figure 4 as a function of the number of participants. The open charm enhancement increases roughly linearly with $\mathrm{N}_{\mathrm{part}}$ reaching a factor $\sim 3$ for central $\mathrm{Pb}-\mathrm{Pb}$ collisions.

\subsection{Final state rescattering}

Lin and Wang ${ }^{7}$ associate the observed excess to D-mesons rescattering in nuclear matter which broadens the $\mathrm{D} \overline{\mathrm{D}} P_{T}$ distribution, leading to an enhancement in the limited phase space of the NA50 experiment. The $\mathrm{D}$ and $\overline{\mathrm{D}}$ scattering is described by a thermal distribution depending on a temperature parameter $T$. This model has been compared to our data. The enhancement factor in the NA50 phase space is calculated as the ratio of the number of dimuons observed at temperature $T$ and $T=0$. A temperature 
of about $200 \mathrm{MeV}$ is needed to reproduce the measured open charm enhancement. Then the shape of the dimuon mass distributions from DD decays is calculated with the corresponding temperature and the normalization obtained from a fit to the mass spectra. Results are shown in figure 5 for $\mathrm{Pb}-\mathrm{Pb}$ collisions with and without final state $\mathrm{D}-$-mesons rescattering. One can see that the rescattering model does not reproduce the experimental mass distribution.

\subsection{Thermal dimuon production}

Other models ${ }^{8,9}$ associate the excess to thermal dimuon production from an expanding fireball. Figure 6 shows the result from an analysis based on the model developped by Rapp and Shuryak ${ }^{8}$ for a central $\mathrm{Pb}-\mathrm{Pb}$ collision. The $\mathrm{D} \overline{\mathrm{D}}$ normalization is deduced from the $\mathrm{D} \overline{\mathrm{D}} / \mathrm{DY}$ proton ratio and the thermal yield is given by the model. We can see that the intermediate mass dimuon production can be well accounted for by thermal radiation when combined with DY and DD productions.

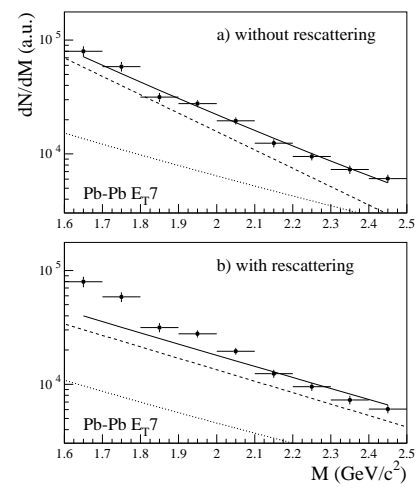

Figure 5: $\mathrm{Pb}-\mathrm{Pb}$ signal mass distributions compared with distributions calculated a) without and b) with $\mathrm{D}$ rescattering.

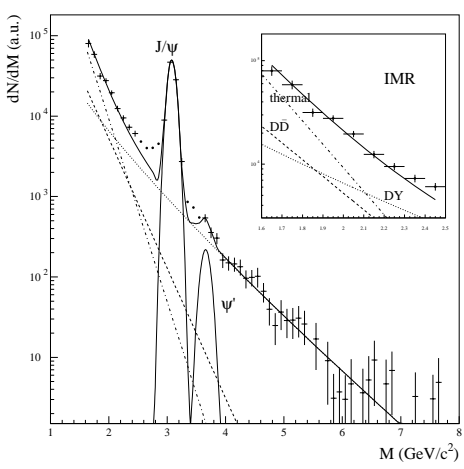

Figure 6: Dimuon mass spectrum from central $\mathrm{Pb}-\mathrm{Pb}$ collisions $\left(\mathrm{N}_{\text {part }} \simeq 380\right)$ compared with the prediction of the thermal $\operatorname{model}^{8}$.

\section{Conclusions}

A 4-Dimensional deconvolution method has been applied to the data collected in $\mathrm{p}-\mathrm{A}, \mathrm{S}-\mathrm{U}$ and $\mathrm{Pb}-\mathrm{Pb}$ collisions. A reasonable value of the open charm cross section has been deduced from proton-nucleus analysis in the intermediate mass region $1.6<M<2.5 \mathrm{GeV} / \mathrm{c}^{2}$. In this mass region, the ion data are in excess of the superposition of DY and DD extrapolated from proton induced collisions. We have shown that the rescattering model of Lin and Wang does not reproduce our data. Finally, we find that the observed excess in heavy ion collisions can be explained assuming either an enhancement of charm production or the presence of thermal dilepton radiation. To distinguish between these two possible explanations, better measurements ${ }^{10}$ are needed.

\section{References}

1. M.C. Abreu et al. (NA38 and NA50 Coll.), Eur. Phys. J C14, 443 (2000).

2. M.C. Abreu et al. (NA50 Coll.), Nucl. Instrum. Methods A 405, 139 (1996).

3. M.C. Abreu et al. (NA50 Coll.), Phys. Lett. B 410, 327 (1997).

4. T. Sjostrand, Comp. Phys. Commun. 82, 74 (1994).

5. M.C. Abreu et al. (NA51 Coll.), Phys. Lett. B 438, 35 (1998).

6. C.Y. Wong and Z.Q. Wang, Phys. Lett. B 367, 50 (1996).

7. Z. Lin and X.N. Wang, Phys. Lett. B 444, 245 (1998).

8. R. Rapp and E. Shuryak, Phys. Lett. B 473, 13 (2000).

9. K. Gallmeister, B. Kämpfer and O.P. Pavlenko, Eur. Phys. J C8, 473 (1999).

10. NA60 Coll., CERN/SPSC 2000-010, Proposal. 